\title{
Blobs in the Winds of Wolf-Rayet Stars
}

\author{
C. Robert, L. Drissen and A. F. J. Moffat, \\ Département de Physique, Université de Montréal.
}

Abstract. Concentrations of material have been observed to propagate in the winds of many W-R stars. These are short-lived, stochastic features of unknown origin.

\section{1) High precision polarimetry}

Thomson scattering is the main process responsible for the linear polarization in the dense and highly ionized winds of W-R stars. A net, non-zero level of polarization results as a consequence of an asymmetric distribution of the scatterers (electrons) or light source(s).

Linear polarization data (with an average rms error of $\pm 0.015 \%$ ) have been obtained since 1984 for a sample of about $30 \mathrm{~W}-\mathrm{R}$ stars (St.-Louis et al. 1987, 1988; Drissen et al. 1987; Robert et al. 1988a, b). All these objects display random polarimetric variations with a typical timescale of a day or less (after subtracting off the periodic modulation in the case of a binary system). Figure 1 shows a clear correlation between the polarization scatter and the spectral subtype especially if one separates WN and WC. Cooler, slow-wind stars of late subtypes (especially WN8 and WC9) show the strongest variations, while hotter, fast-wind stars are quieter.

Blobs of dense material are proposed to be propagating outwards in the winds of all W-R stars. These blobs are formed less frequently, are partially destroyed or have less time to grow in the case of the faster stellar winds (Drissen et al. 1987; Robert et al. 1988a).

\section{2) High resolution spectroscopy}

High signal-to-noise (300-500) and high resolution (0.1 $\AA$ ) spectra have revealed small bumps particularly in the CIII $5696 \AA$ line of the WC stars WR 135,137 and 140 , and in the HeII $5411 \AA$ line of the WN stars WR 134 and WR 136 (Moffat et al. 1988).

Typically, the bumps appear randomly in time, at different velocities and fade away on a timescale of several hours. Difference spectra of WR 135 (Figure 2) show that the bumps that are red-shifted relative to the line center become even more red-shifted with time, while blue-shifted bumps become more blue-shifted with time.

These small structures are interpreted as blobs (as seen independantly in polarization?) of emitting wind material accelerated outwards along with the general wind.

\section{3) Origin}

The origin of the blobs is not known. Non-radial pulsations are not believed to develop in W-R stars (Matthews and Beech 1987; Cox and Cahn 1988; however see Noels and Scuflaire 1987). The theory of vibrational instabilities (Maeder 1985) predicts a correlation of the dispersion with spectral subtype which is opposite to that observed in Figure 1. A phenomenon related to fast rotation is unlikely, according 
to the spectroscopic behavior of the blobs. Radiatively driven instabilties appear to be the most promising explanation. However, a simple model based on the growth rate of the amplitude of the perturbations (Owocki, Castor and Rybicki 1988) in the context of this theory fails to reproduce the trend in Figure 1, which is also seen in continuum light variations (Lamontagne and Moffat 1987).
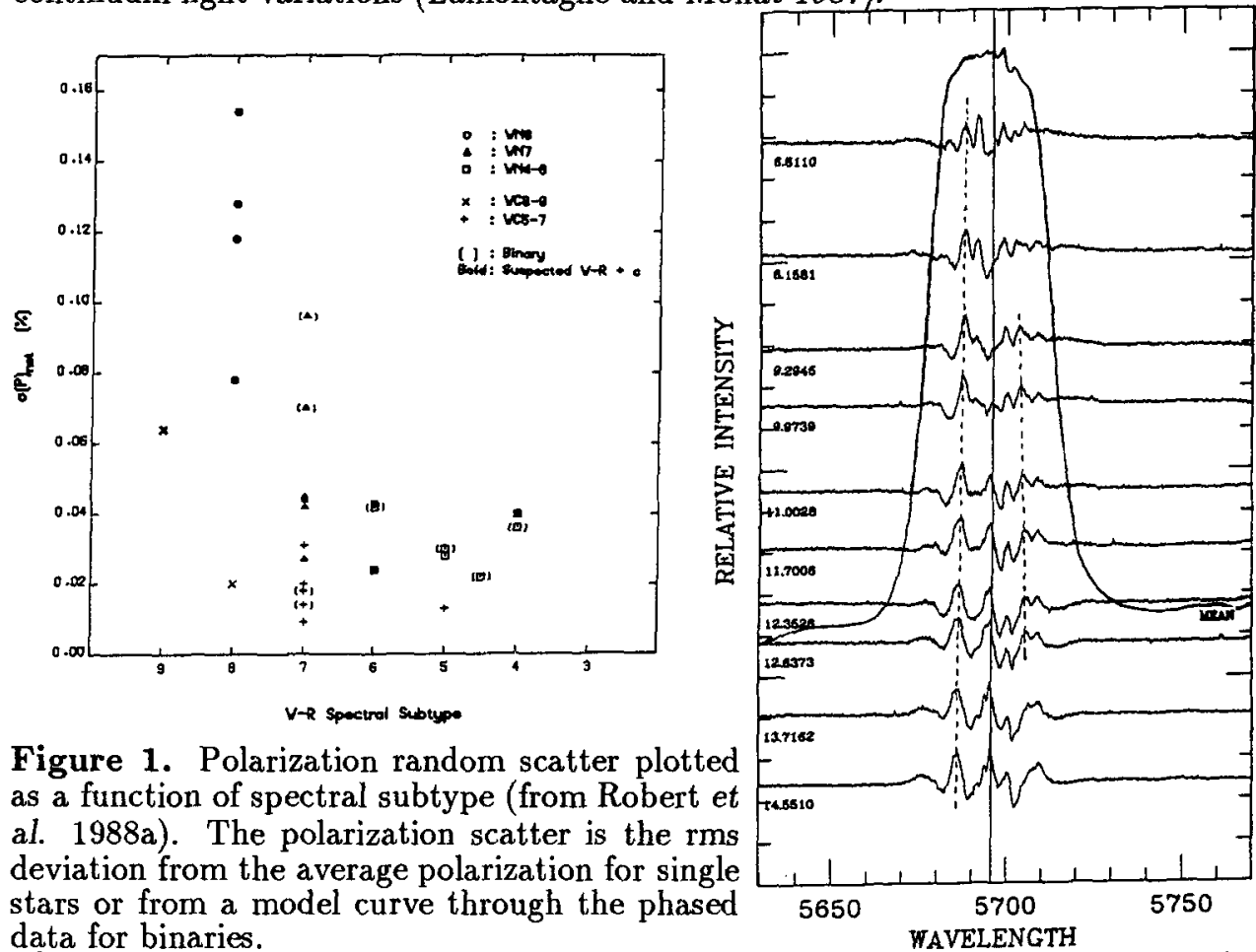

Figure 1. Polarization random scatter plotted as a function of spectral subtype (from Robert et al. 1988a). The polarization scatter is the rms deviation from the average polarization for single stars or from a model curve through the phased data for binaries.

WAVELENGTH

Figure 2. Difference from the mean of spectra for WR 135 obtained on July $6 / 7$, 1988 at Canada France Hawaii Telescope. The mean spectrum (same scale) is the average of all spectra collected during the four night run. The UT time is given for each observation. The vertical line indicates the mean central wavelength. Dashed lines show the radially outwards acceleration of two sample blobs. Note that the dips are not real; they are an artifact of the subtraction method.

\section{References:}

Cox, A. N., and Cahn, J. H. 1988, Ap. J., 326, 804.

Drissen, L., St.-Louis, N., Moffat, A. F. J., and Bastien, P. 1987, Ap. J., 322, 888.

Lamontagne, R., and Moffat, A. F. J. 1987, A. J., 94, 1008.

Maeder, A. 1985, Astr. Ap., 147, 300.

Matthews, J. M., and Beech, M. 1987, Ap. J., 313, L25.

Moffat, A. F. J., Drissen, L., Lamontagne, R., and Robert, C. 1988, Ap. J., 334, 1038.

Noels, A., and Scuflaire, R. 1987, in Instabilities in Luminous Early Type Stars, ed Lamers and de Loore (Dordrecht: Reidel), p. 213.

Owocki, S. P., Castor, J. I., and Rybicki, G. B. 1988, submitted to Ap. J.

Robert, C., Moffat, A. F. J., Bastien, P., Drissen, L., and St.-Louis, N. 1988a, submitted to Ap. J.

Robert, C., Moffat, A. F. J., Bastien, P., St.-Louis, N., and Drissen, L. 1988b, submitted to Ap. J.

St.-Louis, N., Drissen, L., Moffat, A. F. J., Bastien, P., and Tapia, S. 1987, Ap. J., 322, 870.

St.-Louis, N., Moffat, A. F. J., Drissen, L., Bastien, P., and Robert, C. 1988, Ap. J., 330, 286. 